\title{
Preparation and characterization of site-specific dechlorinating microbial inocula capable of complete dechlorination enriched in anaerobic microcosms amended with clay mineral
}

\author{
Zsuzsanna Nagymáté ${ }^{1}$ Laura Jurecska ${ }^{1} \cdot$ Csaba Romsics $^{1} \cdot$ Fanni Tóth $^{1} \cdot$ Viktória Bódai $^{2}$. Éva Mészáros ${ }^{1,3}$. \\ Attila Szabó ${ }^{1} \cdot$ Balázs Erdélyi $^{2} \cdot$ Károly Márialigeti $^{1}$
}

Received: 31 July 2019 / Accepted: 22 January 2020 / Published online: 3 February 2020

(c) The Author(s) 2020

\begin{abstract}
Short-chain halogenated aliphatic hydrocarbons (e.g. perchloroethene, trichloroethene) are among the most toxic environmental pollutants. Perchloroethene and trichloroethene can be dechlorinated to non-toxic ethene through reductive dechlorination by Dehalococcoides sp. Bioaugmentation, applying cultures containing organohalide-respiring microorganisms, is a possible technique to remediate sites contaminated with chlorinated ethenes. Application of site specific inocula is an efficient alternative solution. Our aim was to develop site specific dechlorinating microbial inocula by enriching microbial consortia from groundwater contaminated with trichloroethene using microcosm experiments containing clay mineral as solid phase. Our main goal was to develop fast and reliable method to produce large amount $(100 \mathrm{~L})$ of bioactive agent with anaerobic fermentation technology. Polyphasic approach has been applied to monitor the effectiveness of dechlorination during the transfer process from bench-scale $(500 \mathrm{~mL})$ to industrial-scale $(100 \mathrm{~L})$. Gas chromatography measurement and T-RFLP (Terminal Restriction Fragment Length Polymorphism) revealed that the serial subculture of the enrichments shortened the time-course of the complete dechlorination of trichloroethene to ethene and altered the composition of bacterial communities. Complete dechlorination was observed in enrichments with significant abundance of Dehalococcoides sp. cultivated at $8{ }^{\circ} \mathrm{C}$. Consortia incubated in fermenters at $18{ }^{\circ} \mathrm{C}$ accelerated the conversion of TCE to ethene by $7-14$ days. Members of the enrichments belong to the phyla Bacteroidetes, Chloroflexi, Proteobacteria and Firmicutes. According to the operational taxonomic units, main differences between the composition of the enrichment incubated at $8{ }^{\circ} \mathrm{C}$ and $18{ }^{\circ} \mathrm{C}$ occurred with relative abundance of acetogenic and fermentative species. In addition to the temperature, the site-specific origin of the microbial communities and the solid phase applied during the fermentation technique contributed to the development of a unique microbial composition.
\end{abstract}

Electronic supplementary material The online version of this article (https://doi.org/10.1007/s11274-020-2806-7) contains supplementary material, which is available to authorized users.

Zsuzsanna Nagymáté

zsuzsanna.nagymate@ttk.elte.hu

1 Department of Microbiology, ELTE - Eötvös Loránd University, Pázmány Péter sétány 1/C, 1117 Budapest, Hungary

2 Fermentia Ltd, Berlini u. 47-49, 1045 Budapest, Hungary

3 Institute of Agricultural Sciences, ETH Zurich, 8315 Lindau, Switzerland 


\section{Graphic abstract}

\section{Development and scale-up of dechlorinating enrichments}

\section{Conditions:}

$\mathrm{T}: 8^{\circ} \mathrm{C}$

pH: 6.5-7

e-donors: lactate, $\mathrm{H}_{2}$ e-acceptors: TCE without shaking

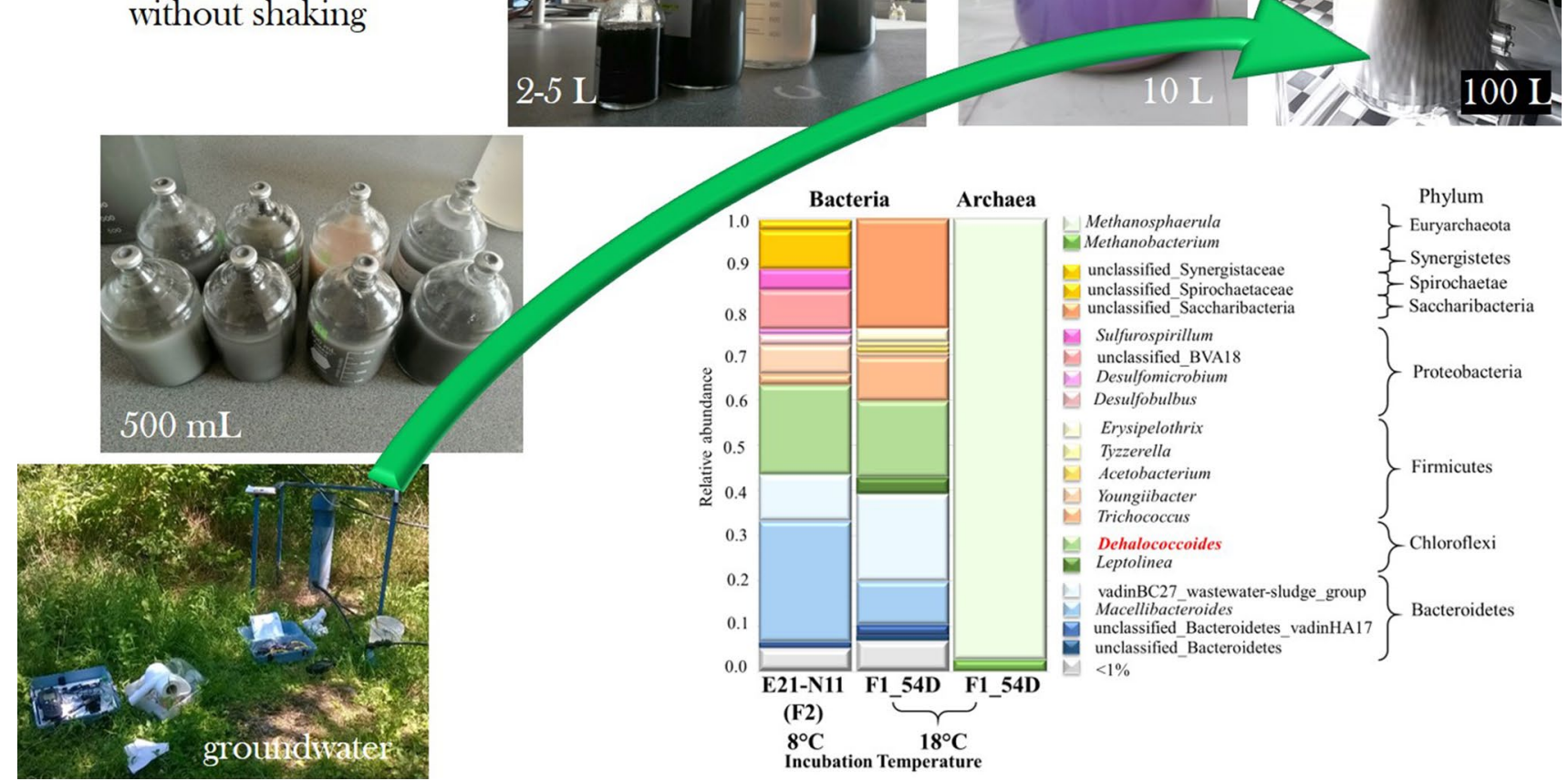

Keywords Dechlorination $\cdot$ Dehalococcoides sp. $\cdot$ Enrichment $\cdot$ Bioaugmentation $\cdot$ Reductive dehalogenase genes

\section{Introduction}

Short-chain halogenated aliphatic hydrocarbons (e.g.: tetrachloroethene-PCE, trichloroethene-TCE) are among the most toxic environmental pollutants of the twentieth century industry (Löffler et al. 2013b). These pollutants can accumulate in the environment causing contamination persisting for decades. TCE can be degraded under anaerobic conditions to non-toxic ethene via cis-dichloroethene (cis-DCE) and vinyl-chloride (VC) through the reductive dechlorination by organohalide-respiring microorganisms (McCarty 1997; Löffler et al. 2013b). Several bacteria are known to have the capability of reductive dechlorination, however, most of the isolated microorganisms can degrade PCE and TCE only to cis-DCE as Desulfitobacterium sp. (Gerritse et al. 1996), Desulfomonile tiedjei (Mohn and Tiedje 1990), Dehalobacter restrictus (Holliger et al. 1998), Desulfuromonas chloroethenica (Krumholz 1997), Sulfurospirillum multivorans (Luijten et al. 2003) and Geobacter sp. (Sung et al. 2006). Thus far, Dehalococcoides sp. had been identified as the only group of microorganisms capable of the complete dechlorination of chlorinated ethenes to non-toxic ethene, via the subsequent reduction of cis-DCE and VC (MaymóGatell et al. 1997; Adrian et al. 2007; Löffler et al. 2013a, b).

Reductive dechlorination can occur as natural attenuation or as a controlled approach such as bioaugmentation at sites contaminated with chlorinated hydrocarbons. In situ biological remediation is a feasible method to degrade shortchain halogenated hydrocarbons, however, microorganisms involved in dechlorination are usually underrepresented or absent in the contaminated areas (Löffler and Edwards 2006; Taş et al. 2010). Therefore, stimulating the autochthonous dechlorinating microbial population (biostimulation; i.e.: adding electron donors and/or nutrients) involved in the 
degradation or using dechlorinating inocula (bioaugmentation) containing Dehalococcoides sp. capable of complete dechlorination, provides a unique solution for remediation of contaminated sites. However, bioaugmentation process requires high cell density, upto $10^{12}$ Dehalococcoides cells $\mathrm{L}^{-1}$ (Waller et al. 2005; Duhamel and Edwards 2006; Holmes et al. 2006; Hug et al. 2012; Delgado et al. 2014; Wen et al. 2017; Saiyari et al. 2018) in active cultures and the transformation of cis-DCE and VC, avoiding the accumulation of the nascent DCE and VC in the environment (Holmes et al. 2006). To establish dechlorinating enrichments, microbial communities were collected and enriched from different anaerobic sites contaminated with PCE and TCE (Duhamel et al. 2002; Holmes et al. 2006), and moreover from sediment of river and tropical mangrove and garden soils not exposed to contamination (He et al. 2005; Ziv-El et al. 2011; Delgado et al. 2014). The medium composition used for the enrichment and the maintenance of organohalide-respiring communities varied and were supplemented with different electron donors such as methanol and hydrogen in medium KB1 (Duhamel et al. 2002; Duhamel and Edwards 2006), lactate in medium ANAS (Holmes et al. 2006) and butyrate in medium DonnaII (Rowe et al. 2008). The developed cultures were maintained as two phases enrichments containing gas and liquid phases under different conditions in a final volume between 0.4 and $5.7 \mathrm{~L}$, residence time between 16 days and 2 years, stirring and the temperature applied varied between 20 and $30^{\circ} \mathrm{C}$ (Hug et al. 2012) coinciding the optimal temperature $\left(25-40{ }^{\circ} \mathrm{C}\right)$ of Dehalococcoides sp. (Taş et al. 2010). Different strains of Dehalococcoides with unique reductive dehalogenase (RDase) genes were enriched (Duhamel et al. 2002; Waller et al. 2005; Duhamel and Edwards 2006; Holmes et al. 2006; Hug et al. 2012) according to the composition of the active cultures.

Bioaugmentation agents apply allochthonous microbial consortia for bioremediation of different sites contaminated with chlorinated aliphatic hydrocarbons. Our aim was to develop a bioremediation technique with the application of site specific autochthonous bioaugmentation agent. Microbial communities enriched from contaminated sites can be applied as site specific bioaugmentation cultures providing an effective strategy to enhance bioremediation processes. The application of predominately autochthonous microbial community adapted to certain pollution and environmental conditions has a greater chance of being effective. The main goal of our work was to produce a site specific bioaugmentation culture using enrichments established in microcosm experiments, and to develop a fast and reliable enrichment method to achieve appropriate inoculum quantity. For this purpose, dechlorinating microbial consortia were collected from PCE and TCE contaminated groundwater. The collected microbial communities were enriched by anaerobic microcosm experiments containing clay mineral as solid phase. Optimization of the transfer process of the anaerobic microcosms at bench- $(500 \mathrm{~mL}, 2 \mathrm{~L}, 5 \mathrm{~L}$ and $10 \mathrm{~L})$ and industrial-scale (in $100 \mathrm{~L}$ working volume fermenter) was the main target of our experiments. Considering the incubation temperature $\left(20^{\circ} \mathrm{C}\right.$ to $\left.30^{\circ} \mathrm{C}\right)$ of the developed dechlorinating microbial cultures, which exceeded the temperature of the groundwater that is below $20^{\circ} \mathrm{C}$. Enrichment of organohalide-respiring microbial communities need to be adapted to temperature comparable to groundwater.

In our work, we focused on the following points of scientific interest. We intended to explore in depth the microbial communities including the composition of Dehalococcoides sp. strains and varieties among the reductive dehalogenase genes playing a role in dechlorination process. Next, the effects of the inoculation and serial transfers upto $100 \mathrm{~L}$ were investigated: the composition of the microbial community and the diversity of Dehalococcoides sp. and thereby the diversity of the key dehalogenase genes impacting the progress of dechlorination activities were monitored throughout the process. Finally, we examined the correlation between the serial transfers and the changes in the composition of the organohalide-respiring bacterial communities and its effect on the dechlorination process. Polyphasic approach was applied to answer these questions. The effectiveness of the dechlorination was monitored based on the concentration change of the intermediate products of the TCE dechlorination by gas chromatography measurement. The bacterial community composition of the groundwater and its changes in enrichments were assessed by Terminal Restriction Fragment Length Polymorphism (T-RFLP) analysis based on 16S rRNA gene over the time. To reveal the composition of the Dehalococcoides sp. strains, 16S rRNA gene and three RDase genes such as trichloroethene (tceA) and vinylchloride reductase ( $v c r A, b v c A)$ genes were sequenced. Next generation sequencing was applied to get a comprehensive insight into the microbial community of enrichments established in $100 \mathrm{~L}$ volume.

\section{Materials and methods}

\section{Samples}

Groundwater samples were collected from two sites (I and II) contaminated with halogenated aliphatic hydrocarbons. The main contaminants were PCE and TCE leaked from storage tanks. Biostimulation was applied beforehand at site I, involving injection of organic substrates into wells. Considering site II no remediation process was carried out previously. Groundwater samples were collected from two wells at each site, sample names refer as follows: $\mathrm{J}$ and $\mathrm{M}$ samples collected from site I; Z and E samples collected from site II (Fig. 1). Groundwater samples were collected 


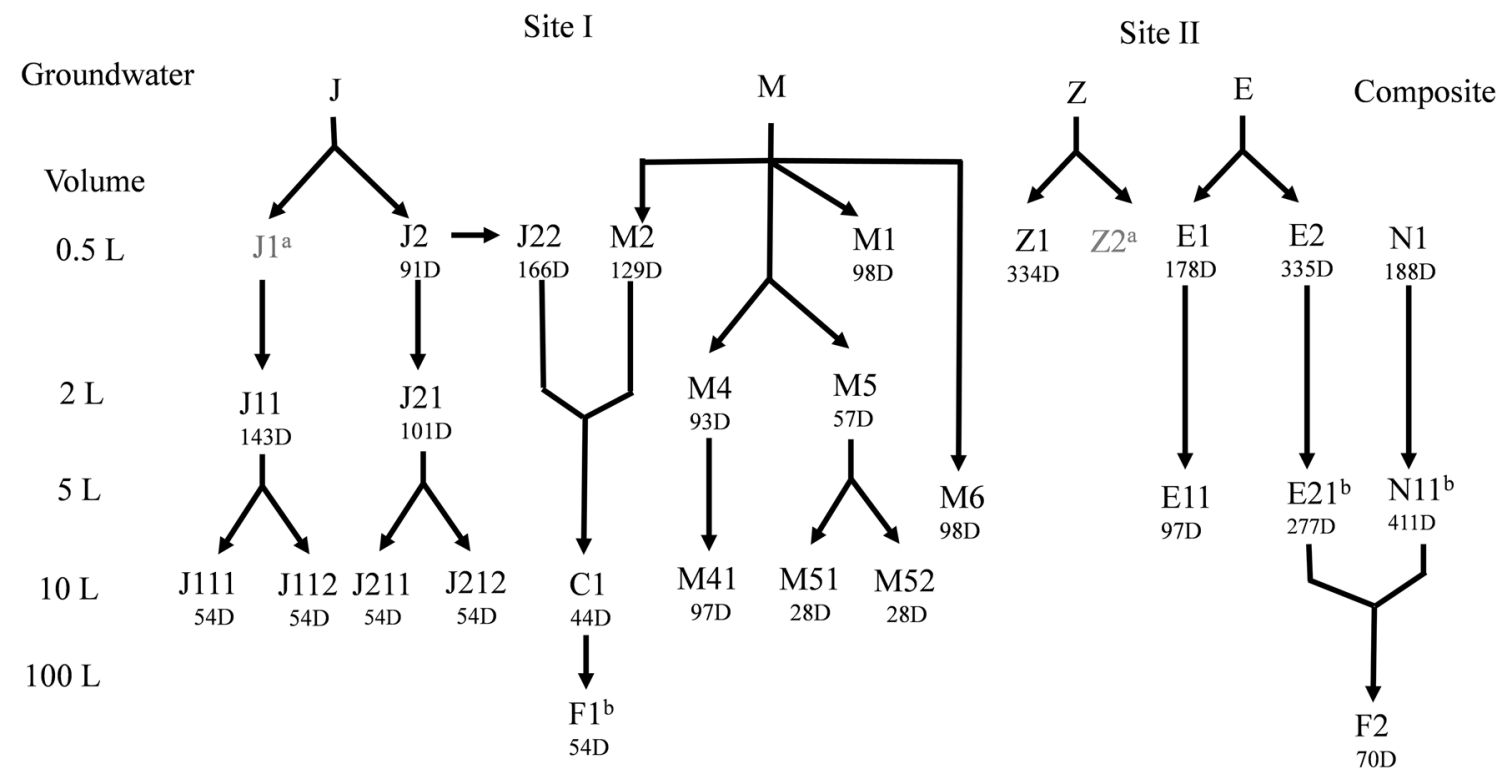

Fig. 1 Sample nomenclature and the scheme of serial transfer procedure of the enrichments. Sampling days are indicated beneath the sample names. Arrows indicate the transfer direction of the enrich-

using low-flow technique in anaerobically sterilized $2.0 \mathrm{~L}$ and $0.5 \mathrm{~L}$ bottles filled up without headspace for microbiological and chemical analysis, respectively. Water samples were kept at $10{ }^{\circ} \mathrm{C}$ during transfer to the laboratory and were processed immediately upon arrival. On site measurement of dissolved oxygen concentration (DO), $\mathrm{pH}$, temperature (T), specific electrical conductivity (EC) and oxidation-reduction potential (ORP) was made using a multimeter (WTW Type Multi 350i, Weilheim, Germany). Water chemistry analysis were performed by the accredited laboratory of Wessling Hungary Ltd (Hungary, Budapest).

\section{Microcosms and enrichment preparation}

Groundwater samples J, M, Z and E were used as inocula to set up enrichments using microcosm experiments. One microcosm group was inoculated with the composite of microbial consortia derived from $\mathrm{J}, \mathrm{E}$ and $\mathrm{Z}$ groundwater samples in ratio 1:1:1 which is referred as N. Five groups of enrichments were established in minimum two parallel (Fig. 1). The enrichments were amended with hydrogen and sour whey containing acetate and lactate as carbon and energy sources to enrich Dehalococcoides sp. and enhance reductive dechlorination. The enrichments prepared in microcosms with $500 \mathrm{~mL}$ volume were transferred into same size bottle $(500 \mathrm{~mL})$ and/or into $2 \mathrm{~L}, 5 \mathrm{~L}$ and $10 \mathrm{~L}$ mesocosms and finally to fermenters with $100 \mathrm{~L}$ volume according to the enrichment scheme shown in Fig. 1. Microcosms were prepared as described by Mészáros et al. (2013). Briefly, the autochthonous microbial community of ments. ${ }^{a}$ No sampling for microbiological analysis; ${ }^{b}$ samples used for next generation sequencing

2.0 L groundwater samples were concentrated on a $0.22 \mu \mathrm{m}$ pore size cellulose nitrate filter (Millipore, Billerica, USA) under $\mathrm{CO}_{2}$ atmosphere to avoid oxygen dissolution during vacuum filtration. The membranes containing the autochthonous microbial communities were shaken in $40 \mathrm{~mL}$ sterile anaerobic medium (Zinder 1998) overnight to produce inocula. Enrichments of different volumes were prepared with the ratio of gas, liquid and solid phases as follows: headspace ratio 1:3; solid to liquid ratio 1:17. The liquid phase of the microrcosms was $425 \mathrm{~mL}$ anaerobic medium (Zinder 1998) purged with 5.0 nitrogen gas (grade 99.99\%) (Linde, Munich, Germany) to maintain anaerobic conditions and $23 \mathrm{~g}$ clay mineral (particle size: $<1.6 \mathrm{~mm}$ ) was used as solid phase. Resazurine $\left(1 \mathrm{~g} \mathrm{~L}^{-1}\right)$ was added to the enrichments as an indicator of the redox condition. Bottles were sterilized for $50 \mathrm{~min}\left(121{ }^{\circ} \mathrm{C}, 1 \mathrm{~atm}\right)$ and the headspace was changed to nitrogen gas. After sterilization $\mathrm{NaHCO}_{3}\left(1 \mathrm{~g} \mathrm{~L}^{-1}\right), \mathrm{Na}_{2} \mathrm{~S}\left(25 \mathrm{mg} \mathrm{L}{ }^{-1}\right)$, vitamins (Zinder 1998), sodium-acetate (2 mM) (Sigma-Aldrich) as carbon and energy source and sour whey (total organic carbon content: $73 \mathrm{~g} \mathrm{~L}^{-1}$ ) containing lactate as carbon source and supplement nutrient were added to the enrichments. The $\mathrm{pH}$ was adjusted to 6.5-7.0 with acetic-acid (5\%). TCE $\left(415 \mu \mathrm{mol} \mathrm{L}^{-1}\right)$ (Sigma-Aldrich, spectrophotometric grade $\geq 99.5 \%$ ) was added as electron acceptor and it was re-dosed after exhausted from the headspace. Furthermore, upon each transfer of enrichments additional TCE was supplied. As last step, enrichments $(500 \mathrm{~mL})$ were inoculated with seed cultures originated from different groundwater samples. The volume of the added inoculum was $10 \% \mathrm{v} / \mathrm{v}$ 
of the liquid phase in each transfer of enrichments. Abiotic control microcosms were set up without any carbon source, electron donors and inocula and were sterilized on three consecutive days. Enrichments were incubated upside down in dark without shaking at $8{ }^{\circ} \mathrm{C}$. Hydrogen gas (grade: $80 \% \mathrm{H}_{2}$ and $20 \% \mathrm{CO}_{2}$ ) (Linde, Munich, Germany) as electron donor (overpressure) was added monthly. Anaerobic fermenter was designed for cultivation of dechlorinating enrichments in $100 \mathrm{~L}$ volume. Fermentation protocol was elaborated for anaerobic inoculation, sampling, feeding and dosage for long term storage. Test run was started to investigate all the required conditions and features throughout the cultivation in the $100 \mathrm{~L}$ prototype fermenter.

\section{The seed train scheme of the enrichments from bench-scale to industrial-scale}

Three different seed trains schemes were applied including two or three transfers in order to achieve appropriate inoculum quantity for the inoculation of anaerobic fermenters with a final volume of $100 \mathrm{~L}$ (Fig. 1). Considering the $10 \% \mathrm{v} / \mathrm{v}$ volume of the inoculum, enrichments inoculated with groundwater $\mathrm{M}$ were established in $2 \mathrm{~L}$ and $5 \mathrm{~L}$ volume, as well (Fig. 1). Enrichments prepared in microcosms with $500 \mathrm{~mL}$ volume and inoculated with the samples of groundwater $\mathrm{J}$ and $\mathrm{E}$ were transferred to $2 \mathrm{~L}$ and $5 \mathrm{~L}$, respectively (Fig. 1). Thereafter enrichments prepared in $2 \mathrm{~L}$ or $5 \mathrm{~L}$ volume were used to inoculate modified Duran laboratory bottles of $10 \mathrm{~L}$ volume or directly to the anaerobic fermenter. Two anaerobic fermenters were inoculated with composite enrichments applying transfer processes as shown in Fig. 1. Two anaerobic fermenters (F1 and F2) were inoculated with the enrichment $\mathrm{C} 1$ prepared in $10 \mathrm{~L}$ volume and the composite of enrichments E21 and N11 prepared in 5-5 L volume (Fig. 1). Enrichments were used as seed culture after TCE had been dechlorinated into cis-DCE and to ethene.

\section{Analytical methods}

The dynamic characteristic of the dechlorination sequence were monitored by using a HP5890 gas chromatograph (Agilent Technologies Inc., Santa Clara, CA, USA) with flame ionization detection equipped with HP-PLOT Q type column $(15 \mathrm{~m} \times 0.53 \mathrm{~mm})$. The method is suitable to separate methane, ethene, vinyl-chloride, cis- and trans-DCE, TCE and PCE providing information on the dominant daughter products of TCE dechlorination appearing in the headspace of the enrichments. The carrier gas was Grade 5.0 helium (Linde, Munich, Germany). The injector was operated in split mode at a ratio 1:20. The temperature protocol was as follows: $60{ }^{\circ} \mathrm{C} 2 \mathrm{~min}, 25^{\circ} \mathrm{C} \mathrm{min}{ }^{-1}$ to $250{ }^{\circ} \mathrm{C}$, the temperature of the detector and the injector was $250{ }^{\circ} \mathrm{C}$. The calibration standards were prepared with same phase ratios and volumes as the microcosms and were stored and sampled in the same way. The injected volume of the headspace was $100 \mu \mathrm{L}$ during manual injection using gas-tight Hamilton (Reno, USA) syringe. Before gas-chromatography measurements enrichments were incubated for $24 \mathrm{~h}$ at $20^{\circ} \mathrm{C}$ to develop the equilibrium of the volatile compounds among the three phases.

Total organic carbon content (TOC) was measured applying a Multi N/C 2100S TC/TN analyser (Analytik Jena, Germany).

\section{Nucleic acid extraction}

For the extraction of the community DNA $2.0 \mathrm{~L}$ of contaminated groundwater was filtered through $0.22 \mu \mathrm{m}$ pore size cellulose nitrate filter (Millipore, Billerica, USA). Before the transferring procedure $2 \mathrm{~mL}$ enrichment samples were collected using a sterile syringe purged with nitrogen gas. Enrichment samples were centrifuged at $12,000 \times g$ for $5 \mathrm{~min}$. DNA was extracted from membrane filters and from the pellets derived from groundwater and enrichments, respectively, using Ultraclean ${ }^{\mathrm{TM}}$ Soil DNA Isolation Kit (MoBio Laboratories Inc. Carlsbad, USA) according to manufacturer's instructions, with a modification at the physical cell disruption step. The samples were shaken in "Bead Solution tubes" (MoBio Laboratories) containing microbeads at $25 \mathrm{~Hz}$ for 2 min using mixer mill MM301 (Retsch, Haan, Germany).

\section{Taxon-specific 16S rRNA and reductive dehalogenase (RDase) genes targeted PCR}

Taxon-specific 16S rRNA gene was used to detect the presence of organohalide-respiring microorganisms, such as Dehalococcoides sp., Desulfitobacterium dehalogenans, Dehalobacter restrictus, Desulfomonile tiedjei, Desulfuromonas chloroethenica and Geobacter sp. Dehalococcoides sp. strains related RDase genes such as trichloroethene reductase (tce A) and vinyl-chloride reductases ( $\mathrm{vcrA}$ and $b v c A$ ) also amplified by PCR. The applied primer sets are presented in Table S1. Dehalococcoides sp. specific 16S rRNA gene sequences were amplified in nested PCR approach as described by Révész et al. (2006). Thermal profiles of the PCR reactions were as follows: initial denaturation at $98{ }^{\circ} \mathrm{C}$ for $5 \mathrm{~min}$, followed by 32 amplification cycles of $30 \mathrm{~s}$ at $94{ }^{\circ} \mathrm{C}$, for $45 \mathrm{~s}$ at target sequence-specific annealing temperature as follows: Dehalococcoides sp. and verA at $55{ }^{\circ} \mathrm{C}, D$. restrictus and $D$. dehalogenans at $65{ }^{\circ} \mathrm{C}, D$. chloroethenica and D. tiedjei at $64^{\circ} \mathrm{C}$, Geobacter sp., tceA and $b v c A$ at $59^{\circ} \mathrm{C}, 49^{\circ} \mathrm{C}$ and $52^{\circ} \mathrm{C}$, respectively, $1 \mathrm{~min}$ at $72{ }^{\circ} \mathrm{C}$, followed by final extension at $72{ }^{\circ} \mathrm{C}$ for $10 \mathrm{~min}$. The PCR reaction mixture contained $1 \mathrm{U}$ of LCTaq DNA Polymerase (Thermo Fisher Scientific Inc., Waltham, MA, USA), 
$200 \mu \mathrm{M}$ of each deoxynucleoside triphosphate, $1 \times$ Taq buffer with $\left(\mathrm{NH}_{4}\right)_{2} \mathrm{SO}_{4}$ (Thermo Fisher Scientific Inc. USA), $2 \mathrm{mM} \mathrm{MgCl} 2,0.32 \mu \mathrm{M}$ of each primer, and 1-2 $\mu \mathrm{L}$ DNA template in final volume of $50 \mu \mathrm{L}$.

\section{T-RFLP analysis}

The bacterial community composition and their changes in enrichments and in fermenters were assessed by T-RFLP analysis over the time. Bacteria 16S rRNA gene was amplified using 27F forward primer labelled with 5'-hexachlorofluorescein and 519R reverse primer (Lane 1991). T-RFLP analysis was performed as described by Nagymáté et al. (2016) with the differences that AluI and Bsh1236I (Thermo Fisher Scientific Inc., USA) endonucleases applied. Electropherograms were analysed with the GeneMapper software (Applied Biosystems; Version 3.7). Fragment size longer than 50 base pairs and fluorescence intensity higher than 50 fluorescence units (peak height) were used to define T-RF peaks. Data matrices were created with T-RF's $\geq 1 \%$ relative abundance using T-RFLP Analysis Expedited (T-REX) software (Culman et al. 2009). Statistical data analysis was performed by Principal Component Analysis (PCA) using PAST software system (Hammer et al. 2001). To verify the significance of the separation among the microbial community of the different enrichment groups considering the used inoculum and the transferring steps, permutational multivariate analysis of variance (PERMANOVA, 999 permutations) was applied using PAST software package.

\section{Sequence analyses}

The 16S rRNA gene fragment of genus Dehalococcoides and the three RDase genes (tceA, vcrA and $b v c A$ ) were sequenced by Sanger method using group specific forward primers at LGC Ltd. (Berlin, Germany). Sequences were aligned by Basic Local Alignment Search Tool (https://www.ncbi. nih.gov/BLAST) using the GenBank nucleotide database. Operational taxonomic units (OTUs) were assigned at 95\% and $97 \%$ similarity threshold levels, representing microbial genera and species, respectively, according to Tindall et al. (2010). Sequences obtained in this study were submitted to European Nucleotide Archive (Accession numbers: LR026649-LR026661 for 16S rRNA gene and LR031310LR031336 for reductive dehalogenase genes).

Prior to inoculation of the fermenter F2 composite sample was prepared by mixing enrichments E21 and N11. The microbial communities of the fermenter F1 and the composite enrichment after 54 and 70 days incubation, respectively, were assessed by high throughput pyrosequencing of the V1-V3 region of 16S rRNA gene. Bacteria 16S rRNA gene was amplified using the same universal primer set as for T-RFLP analyses (Nagymáté et al. 2016). Archaea 16S
rRNA gene was amplified using CS1-A519F and CS2-A855R (Klindworth et al. 2013) primer sets with the following thermal conditions: initial denaturation at $98{ }^{\circ} \mathrm{C}$ for $5 \mathrm{~min}$, followed by 25 amplification cycles of $30 \mathrm{~s}$ at $95^{\circ} \mathrm{C}$, for $30 \mathrm{~s}$ at $60{ }^{\circ} \mathrm{C}, 30 \mathrm{~s}$ at $72{ }^{\circ} \mathrm{C}$, followed by final extension at $72{ }^{\circ} \mathrm{C}$ for $10 \mathrm{~min}$. The forward primers were fused with sequencing barcodes and adapters. The amplification and subsequent next generation sequencing of Bacteria and Archaea 16S rRNA genes was performed as described by Krett et al. (2017). Emulsion PCR, amplicon library processing and pyrosequencing were performed on GS Junior sequencing platform according to the Lib-L protocol of the manufacturer (Roche/454 Life Sciences). Bioinformatics analyses of the resulting sequence reads were carried out with mothur (Schloss et al. 2009) as described in detail by Szabó et al. (2017). Sequences were aligned and classified by the SINA v1.2.11 aligner tool (Pruesse et al. 2012) using the ARBSILVA SSU NR reference database-SILVA Release 123 (Quast et al. 2013). Sequences were submitted to the NCBI SRA database and available under the BioSample accession number SAMN10024392.

Representatives of the established operational taxonomic units (OTUs) were selected for in silico determination of the individual T-RF peaks of the community members especially the Dehalococcoides sp. For this purpose, unique T-RF's of the OTUs were identified by MEGA6 program based on the recognition site of restriction endonucleases applied for T-RFLP analysis (AluI and Bsh1236I) (Tamura et al. 2013).

\section{Results}

\section{Physico-chemical and molecular biological data of the two contaminated sites}

Physico-chemical parameters of the groundwater samples are listed in Table 1. The groundwater samples, originated from geographically-distinct sites, were characterized with neutral $\mathrm{pH}$ 6.8-7.4 and comparable temperature ranging 13.1-14.5 ${ }^{\circ} \mathrm{C}$. According to the specific electrical conductivity measurements the groundwater samples showed remarkable differences. Sample from well J was characterized with the highest value $2260 \mu \mathrm{S} \mathrm{cm}^{-1}$ caused by the injection of organic substrates, while the specific electrical conductivity of the other samples was lower ranged in 1031-1260 $\mu \mathrm{S} \mathrm{cm}^{-1}$ (Table 1). The DO concentration was low varying between 0.11 and $1.27 \mathrm{mg} \mathrm{L}^{-1}$ and the oxidation-reduction potential was below $-94 \mathrm{mV}$ (Table 1) at site I indicating prevailing anoxic conditions. Concentration of ammonium- and phosphate-ions were slightly higher in well $\mathrm{E}$, while TOC and nitrate concentrations were higher in well $\mathrm{J}$ and $\mathrm{M}$ (Table 1). Chemical analyses of groundwater 
Table 1 Physico-chemical characteristics of groundwater samples collected from the wells of the contaminated sites; n.d. no data available due to technical failure

\begin{tabular}{|c|c|c|c|c|}
\hline & \multicolumn{2}{|l|}{ Site I } & \multicolumn{2}{|l|}{ Site II } \\
\hline & $\mathrm{J}$ & M & $\mathrm{Z}$ & $\mathrm{E}$ \\
\hline $\mathrm{pH}$ & 7.48 & 7.47 & 6.83 & 8.15 \\
\hline $\mathrm{T}\left({ }^{\circ} \mathrm{C}\right)$ & 14.3 & 14.5 & 13.3 & 14.1 \\
\hline $\mathrm{DO}\left(\mathrm{mg} \mathrm{L}^{-1}\right)$ & 0.5 & 0.3 & 0.1 & 1.3 \\
\hline ORP (mV) & -94 & -458 & n.d & n.d \\
\hline $\mathrm{EC}\left(\mu \mathrm{S} \mathrm{cm}{ }^{-1}\right)$ & 2260 & 1260 & 1022 & 1031 \\
\hline $\mathrm{NH}_{4}{ }^{+}-\mathrm{N}\left(\mathrm{mg} \mathrm{L}^{-1}\right)$ & 1.3 & 0.6 & 0.5 & 2.9 \\
\hline $\mathrm{NO}_{2}^{-}-\mathrm{N}\left(\mathrm{mg} \mathrm{L}^{-1}\right)$ & $<0.01$ & $<0.01$ & $<0.01$ & $<0.01$ \\
\hline $\mathrm{NO}_{3}{ }^{-}-\mathrm{N}\left(\mathrm{mg} \mathrm{L}^{-1}\right)$ & 1.5 & 1.1 & 0.5 & 0.8 \\
\hline $\mathrm{Cl}^{-}\left(\mathrm{mg} \mathrm{L}^{-1}\right)$ & 266 & 72 & 39 & 43 \\
\hline $\mathrm{Fe}\left(\mathrm{mg} \mathrm{L}^{-1}\right)$ & 8.20 & 1.36 & 9.07 & 8.08 \\
\hline $\mathrm{SO}_{4}{ }^{2-}\left(\mathrm{mg} \mathrm{L}^{-1}\right)$ & 6 & 100 & 129 & 74 \\
\hline $\mathrm{PO}_{4}{ }^{3-}\left(\mathrm{mg} \mathrm{L}^{-1}\right)$ & 0.66 & $<0.5$ & 1.68 & 15.1 \\
\hline TOC $\left(\mathrm{mg} \mathrm{L}^{-1}\right)$ & 363 & 400 & 23 & 26 \\
\hline $\operatorname{PCE}\left(\mu \mathrm{g} \mathrm{L}^{-1}\right)$ & $<1$ & $<1$ & $<1$ & 15.6 \\
\hline $\operatorname{TCE}\left(\mu \mathrm{g} \mathrm{L}^{-1}\right)$ & 25 & 47 & 45 & 246 \\
\hline cis-DCE $\left(\mu \mathrm{g} \mathrm{L}^{-1}\right)$ & 58,900 & 1350 & 82 & 187 \\
\hline trans-DCE $\left(\mu \mathrm{g} \mathrm{L}^{-1}\right)$ & $<1$ & 30 & $<1$ & 1 \\
\hline $\mathrm{VC}\left(\mu \mathrm{g} \mathrm{L}^{-1}\right)$ & 66,600 & 390 & 1 & 6 \\
\hline ethene $\left(\mu \mathrm{g} \mathrm{L}^{-1}\right)$ & 970 & 4300 & $<5000$ & $<5000$ \\
\hline methane $\left(\mu \mathrm{g} \mathrm{L}^{-1}\right)$ & 3310 & 14,290 & $<5000$ & $<5000$ \\
\hline $\operatorname{VOCl}\left(\mu \mathrm{g} \mathrm{L}^{-1}\right)$ & 65,373 & 1437 & 128 & 450 \\
\hline
\end{tabular}

The volatile chlorinated hydrocarbons concentration abbreviated as $\mathrm{VOCl}$

revealed high volatile chlorinated hydrocarbons $(\mathrm{VOCl})$ contamination (Table 1) exceeding $65 \mathrm{mg} \mathrm{L}^{-1}$ concentration with main compounds of cis-DCE, VC and traces of TCE at site I. Moreover, methane (3.3-14.2 $\mathrm{mg} \mathrm{L}^{-1}$ ), ethene (0.97-4.3 $\left.\mathrm{mg} \mathrm{L}^{-1}\right)$ and high chloride-ion concentration (72-266 $\mathrm{mg} \mathrm{L}^{-1}$ ) indicated ongoing reductive dechlorination at site I (Table 1). Significantly lower VOCl contamination was revealed at site II with TCE and cis-DCE as main compounds and traces of PCE, trans-DCE and VC (Table 1). Though, ethene concentration was not detected in the wells of the site II indicating partial dechlorination process under natural conditions.

Regarding the targeted detection of microorganisms capable of reductive dechlorination only minor differences were observed among the samples with genera Geobacter, Dehalobacter, Desulfitobacterium, Desulfomonile and Desulfuromonas capable of partially dechlorinated PCE and TCE to cis-DCE (DeWeerd et al. 1990; Utkin et al. 1994; Krumholz 1997). 16S rRNA gene amplicons of Geobacter were detected in all examined groundwater. The sequence analysis of 16S rRNA gene revealed the presence of Geobacter lovleyi strain $\mathrm{SZ}$ in the groundwater. Members of genus
Dehalococcoides were present in all examined groundwater. However, the distribution of Dehalococcoides sp. related RDase genes such as tceA, vcrA and $b v c A$ genes showed differences (Table 2). Amplicons of the three RDase genes were detected in both wells of site I ( $\mathrm{J}$ and $\mathrm{M})$ and in groundwater $\mathrm{Z}$ at site II. Amplicon of tceA gene was not detected in groundwater $E$. The sequence analysis of $16 \mathrm{~S}$ rRNA gene fragment of Dehalococcoides sp. obtained from groundwater revealed $100 \%$ sequence similarity to Dehalococcoides $\mathrm{sp}$. GT, FL2, CBDB1, BAV1 and BTF08 strains like microorganisms. Sequence of tceA and vcrA genes retrieved from sample J revealed the presence of Dehalococcoides sp. BTF08 (Pöritz et al. 2013) and Dehalococcoides sp. UCH007 strains like microorganisms (Uchino et al. 2015), respectively (Table 2). Sequence analysis of $v c r A$ gene obtained from groundwater E and M resulted Dehalococcoides sp. UCH007 and Dehalococcoides sp. BTF08 strains like microorganisms, respectively (Table 2). However, tceA amplicon retrieved from samples $\mathrm{M}$ and $\mathrm{Z}$ and $v c r A$ gene obtained from groundwater $Z$ resulted ambiguous mixed base sequences. The sequence analyses of the $b v c A$ gene confirmed the presence of Dehalococcoides sp. BAV1 strain like microorganism (Table 2) in each groundwater. According to the sequence analyses of the three RDase genes diverse Dehalococcoides population was present in the examined sites.

\section{TCE dechlorination in enrichments}

The enrichments inoculated with microbial community derived from groundwater with different origin had different lag times prior to dechlorination and were characterized with different TCE degradation potential. Dechlorination of TCE with the accumulation of cis-DCE started 15 to 57 days following the establishment of the enrichments in $500 \mathrm{~mL}$ volume.

The enrichments of groups $\mathrm{J}$ and $\mathrm{M}$ maintained in different volume were characterized with the most efficient TCE degradation potential, since TCE was transformed to cis-DCE in less than 30 days (Fig. 2). Partial dechlorination with the accumulation of vinyl-chloride was observed in the transferred (second stage) enrichments $(500 \mathrm{~mL}$ and 2 L; J11, J21 and J22) (Table 2; Fig. 2) showing increased TCE degradation efficiency compared to first stage enrichments prepared in $500 \mathrm{~mL}$. Enrichments J11 and J21 were transferred to $10 \mathrm{~L}$ volume scale (Fig. 1, Table 2) after short-term (101 days-accumulation of cis-DCE) and long-term (493 days-accumulation of ethene) incubation, respectively. Complete dechlorination of TCE, with increasing concentration of cis-DCE, VC and accumulation of ethene, was achieved in the $10 \mathrm{~L}$ volume enrichments by 126 to 291 days. Considering the time-scale of the three-step transferring process from groundwater to $10 \mathrm{~L}$, complete 


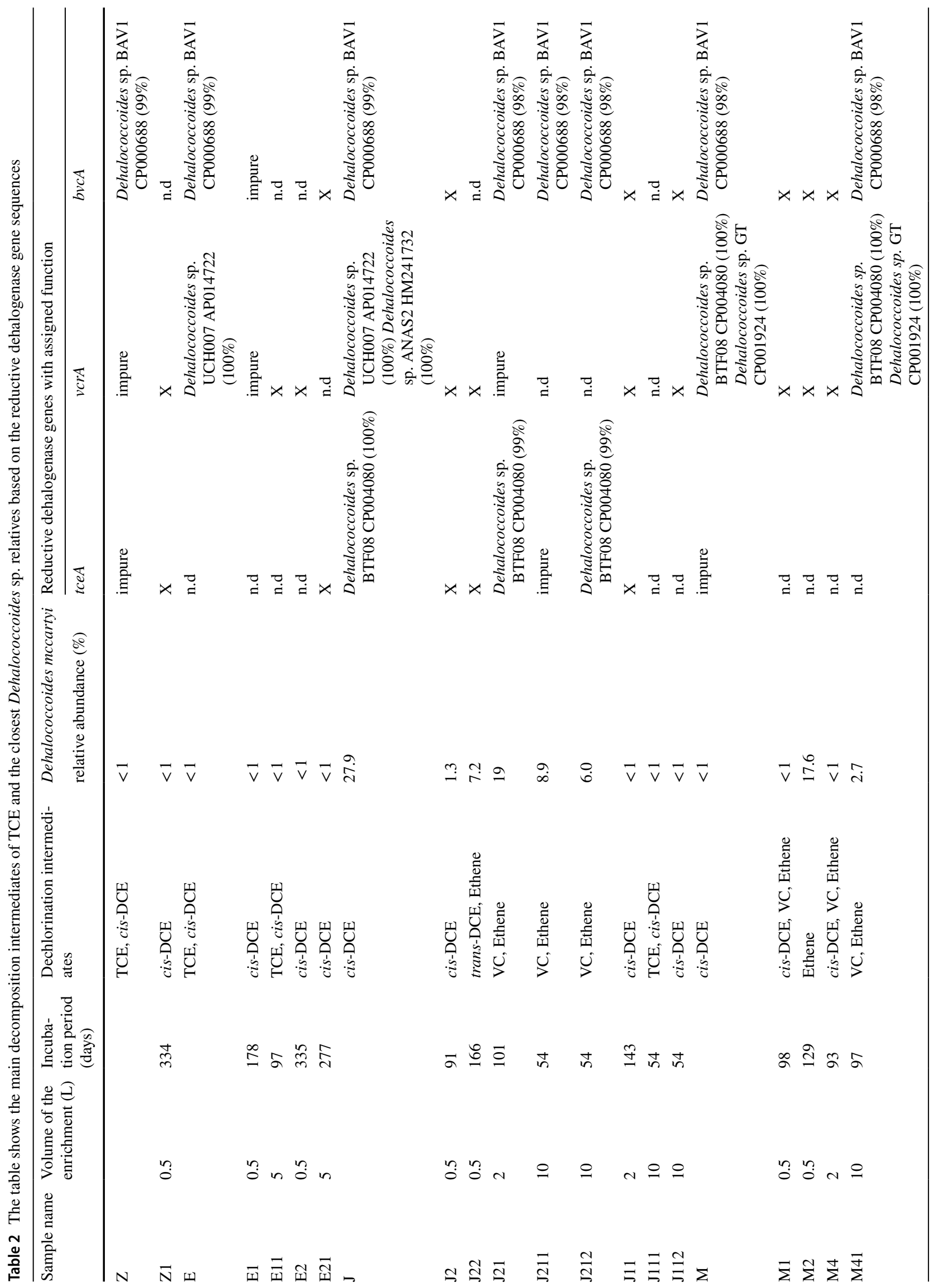




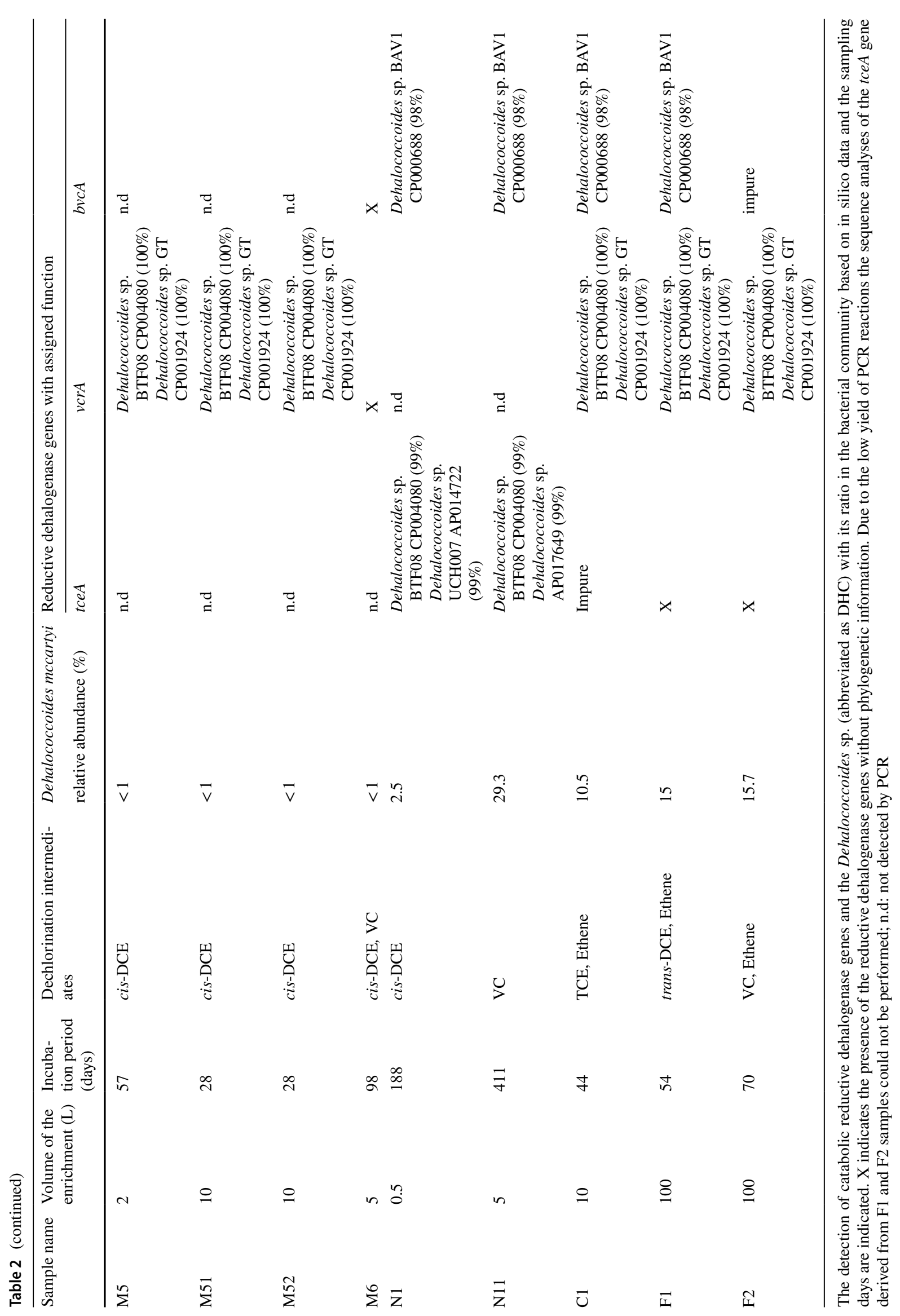



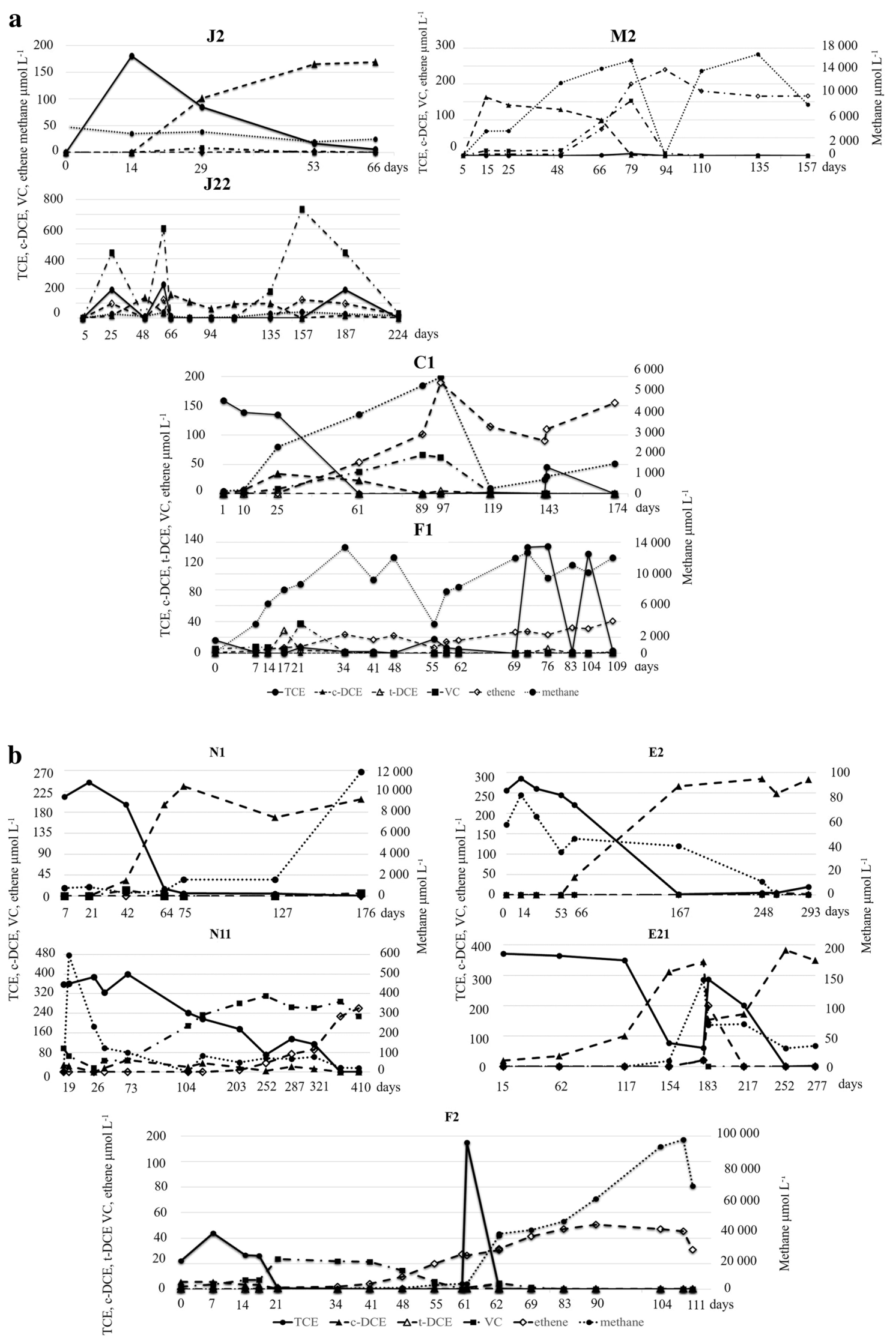
4Fig. 2 TCE transformation to daughter products in the enrichments transferred to industrial scale by two different transfer schemes including two or three transfer steps to achieve bioaugmentation inoculum over time. Inoculation pathways of fermenters F1 (a) and F2 (b)

dechlorination of TCE to ethene was observed in enrichments assigned as J by 392 or 619 days applying short- and long-term incubation, respectively.

Composite enrichments were applied to inoculate fermenters F1 and F2 to shorten the time-scale of dechlorination. Two composite enrichments, $\mathrm{C} 1$ with $10 \mathrm{~L}$ volume and the composite of enrichments N11, E21 with 5-5 L volume, were used to inoculate the fermenters F1 and F2, respectively (Figs. 1, 2). Enrichment $\mathrm{C} 1$ was characterized with accelerated dechlorination since all TCE was transformed in line with increasing ethene concentration by day 61 (Fig. 2). Accelerated dechlorination was observed in the composite enrichment N11 with the accumulation of VC and ethene by day 19 and 252, respectively. However, in the enrichment E21 inoculated with groundwater originated from nontreated site II, TCE was dechlorinated mainly to cis-DCE with the detection of traces of $\mathrm{VC}$ and ethene during the 277 days incubation period. Our results imply fast TCE degradation to cis-DCE and $\mathrm{VC}$, whereas the further dechlorination step with the presence of ethene was slower suggesting co-metabolic VC dechlorination in large scale enrichments. Considering the intermediates of the dechlorination of TCE, differences were observed between the two fermenters. TCE was transformed to ethene with the periodic detection of trans-DCE in F1 in 14 days. While, in the fermenter F2 low amounts of cis-DCE and VC were detected during the TCE conversion to ethene taking only 7 days. Moreover, the parallel headspace measurements showed significant amount of methane (Fig. 2) providing indirect evidence for the presence of methanogenic Archaea.

Conforming the analytical results enrichments maintained capabilities to dechlorinate TCE after the transferring procedures, implying the effectiveness of the applied anaerobic inoculation and fermentation processes. However, based on the gas chromatography measurements, accumulation of the daughter products of the TCE dechlorination was observed, exceeding the intial molar concentration of TCE. Presumably, TCE was adsorbed to the applied solid phase causing lower concentration in the headspace. This phenomenon was further strengthened by periodic addition of TCE causing more extent accumulation of volatile products such as ethene and VC and to a lesser extent cis-DCE in the headspace.

\section{The bacterial community structure in the groundwater and in enrichments}

To assess the changes in the bacterial communities $16 \mathrm{~S}$ T-RFLP analyses were applied throughout the experiment.
The bacterial community of the collected groundwater samples characterized with different physico-chemical parameters showed remarkable separation according to the PCA ordination of 16S T-RFLP fingerprints based on the first two principal components (Fig. 3) suggesting differently structured communities. Based on the first two principal components the enrichments inoculated with microbial communities derived from distinct groundwater samples also showed significant differences $\left(\mathrm{F}_{\text {PERMANOVA }}\right.$ : 2.58; p: 0.0001), furthermore the microbial community of duplicate enrichments showed only slight separation (Fig. 3). Our results imply that the selective enrichment procedure caused significant ( $\mathrm{F}_{\text {PERMANOVA }}: 2.58$; $\mathrm{p}$ : 0.0001 ) changes in the bacterial community composition of enrichments compared to groundwater (Fig. 3). The bacterial community structures were significantly influenced by transferring steps also, especially the third transfer step ( $\mathrm{F}_{\text {PERMANOVA }}: 1.76$; $\mathrm{p}$ : 0.013), presumably related to the length of incubation. Furthermore, according to the presence of $c i s$-DCE and ethene as dominant by-products of TCE dechlorination, significant ( $F_{\text {PERMANOVA }}: 1.65 ; \mathrm{p}$ : 0.018 ) differences were observed in the community structures suggesting the alteration and the slight decrease in the diversity indices (data not shown) of the bacterial community with the progress of dechlorination process regardless of the volume of the enrichments.

\section{Taxon-specific detection and analysis of reductive dehalogenase genes}

Presence of bacteria capable of reductive dechlorination (such as members of genera Geobacter, Desulfitobacterium, Desulfomonile, Desulfuromonas and Dehalococcoides) were assayed in the enrichments after TCE was transformed. The 16S rRNA gene amplicons of Geobacter spp., identical to Geobacter lovleyi strain SZ (100\% sequence similarity), were detected in the enrichments similarly to the groundwater. In all enrichments characterized with partial and complete dechlorination Dehalococcoideslike sequences could be detected by direct and nested PCR approach. Sequence analysis of 16S rRNA gene, revealed two ribotypes of Dehalococcoides in the enrichments. The retrieved sequences were identical (100\% sequence similarity) to Dehalococcoides sp. GT, FL2, CBDB1, BAV1 and BTF08 strains like microorganisms as was observed in the case of the groundwater samples. Another ribotype of Dehalococcoides was obtained from enrichment inoculated with groundwater M showing $100 \%$ similarity to Dehalococcoides mccartyi 195 (T).

Contrary, the detection of Dehalococcoides related RDase genes (tceA, vcrA and $b v c A$ ) showed differences (Table 2). Amplicons of $t c e A$ gene were detected in enrichment groups 
Fig. 3 Two-dimensional principal component analysis (PCA) plot of T-RFLP data retrieved from bacterial communities of groundwater and enrichments. Sampling days are indicated following the sample names (e.g.: M5_57D)

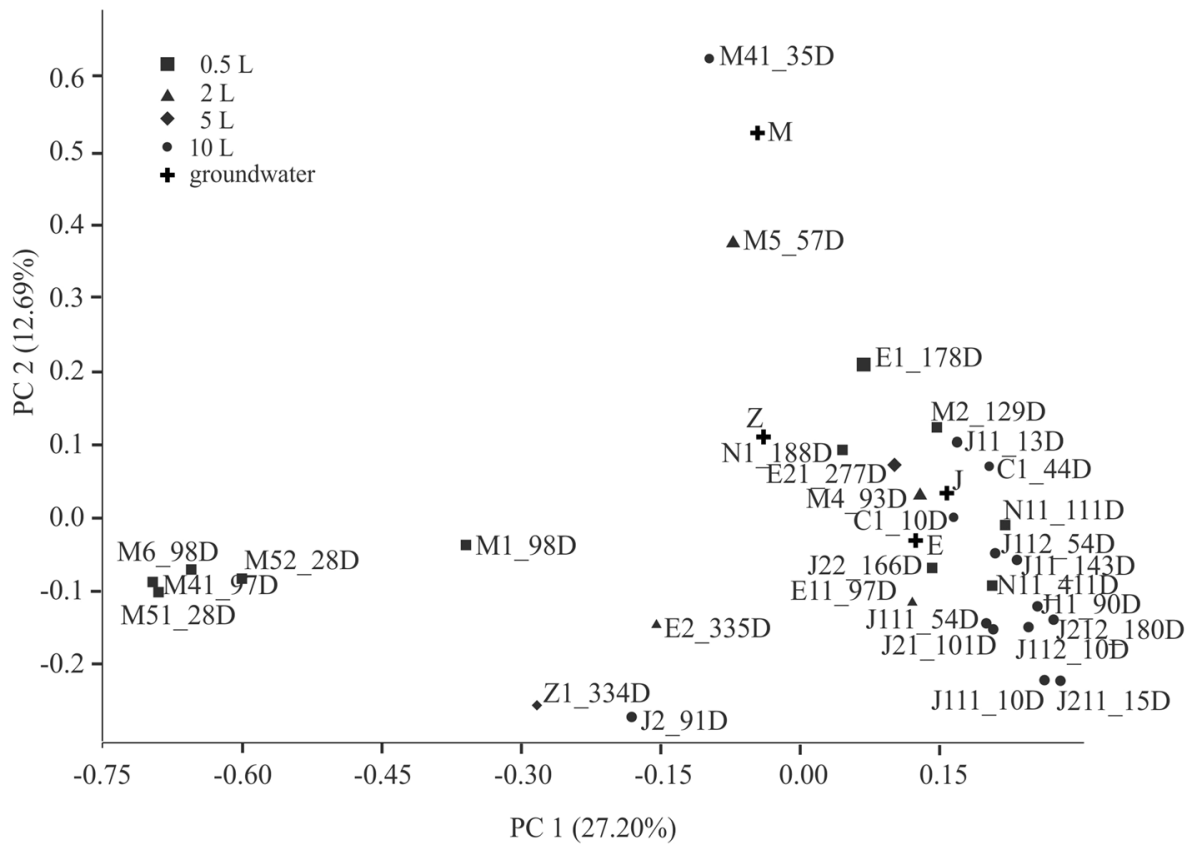

$\mathrm{J}$ and $\mathrm{N}$ predominantly (Table 2 ). Considering the presence of the tceA gene differences were observed in the case of enrichments $\mathrm{M}$ and $\mathrm{E}$. Groundwater $\mathrm{M}$ was characterized with the presence of $t c e A$ gene, which was not detected in the enrichments. In enrichments referred as $E$ the presence of tce $A$ gene was only detected after selective enrichment steps, suggesting low copy number of tceA gene in groundwater $\mathrm{E}$. Both vinyl-chloride reductase genes ( $v c r A$ and $b v c A)$ were detected in enrichments $(500 \mathrm{~mL})$ inoculated with groundwater (Table 2). However, enrichments transferred to $5 \mathrm{~L}$ and $10 \mathrm{~L}$ volumes were usually characterized with the presence of only one vinyl-chloride reductase gene (Table 2). Amplicons of all the three RDase genes were merely detected in two enrichments inoculated with groundwater $\mathrm{J}$ and in the two fermenters suggesting the presence of a non-detectable minor Dehalococcoides sp. population in enrichments. The phylogenetic analyses of tce $A, v c r A$ and $b v c A$ genes revealed the presence of Dehalococcoides sp. BTF08, UHC007, GT and BAV1 strains like microorganisms (Table 2).

\section{NGS results}

$16 \mathrm{~S}$ rRNA gene amplicon sequencing of the fermenter F1 and the composite sample of enrichments E21 and N11 used for inoculation of the fermenter F2 resulted in 8650 and 1492 high-quality reads, respectively, classified within the Bacteria domain. Rarefaction curves completely reached a plateau suggesting that the sequencing depth was appropriate to cover the bacterial diversity (data not shown). The diversity estimators including ACE (F1: 106; E21-N11: 42), Chao1 (F1: 106; E21-N11: 42), Shannon-Wiener (F1: 2.4; E21-N11: 2.51) and Simpson's (F1:
0.14; E21-N11: 0.12) indices implied low diversity of the bacterial community. Common members of the enrichments belonged to the phyla Bacteroidetes, Chloroflexi, Proteobacteria, Firmicutes, Spirochaetae and Synergistetes (Fig. 4). Bacteroidetes and Chloroflexi populations were the most dominant members of both cultures. Significant prevalence of phylum Saccharibacteria was also observed in the bacterial community of the fermenter F1. Representatives of phyla Spirochaetae and Synergistetes were solely identified from the composite enrichment (E21-N11) used for the inoculation of fermenter F2. The organohalide-respiring genus Dehalococcoides with relative abundance of $19.83 \%$ and $16.98 \%$ gave the majority of the microbial community of composite enrichment and fermenter F1, respectively (Fig. 4). In the composite enrichment (E21-N11) other putative dechlorinators as Sulfurospirillum sp. and Geobacter sp. were present with low relative abundance of 4.5 and $<1 \%$, respectively (Fig. 4). Sulfurospirillum sp., Geobacter sp. and Dehalobacterium sp. as putative dechlorinators were also detected in the fermenter F1, however, their relative abundance did not exceed the $1 \%$. In addition to the genus Dehalococcoides other four abundant OTUs belonging to mainly presumed fermentative (Macellibacteroides spp., Trichococcus spp., Youngibacter spp.) and amino-acid degrading bacteria (vadinBC_27_wastewater-sludge_group) were shared among the two enrichments (Fig. 4). Dehalocococcides sp. and the fermentative bacteria represented the core bacterial community of both enrichments. Presumed acetogenic bacteria (Acetobacterium spp., Tyzzerella spp., Desulfobacter spp., Desulfobulbus spp., Desulfomicrobium spp. and Desulfuromonadales) with considerably 
different relative abundances (Fig. 4) were also detected in the enrichments. However, the composition of acetogenic species differed in the two enrichments. Among the acetogens a variety of sulphate-reducing species were detected. According to the Archaea 16S rRNA gene amplicon sequencing of the fermenter F1 Methanobacterium and Methanosphaerula were identified with the significant prevalence of Methanosphaerula (relative abundance 97\%) (Fig. 4).

\section{In silico analysis}

Based on the in silico 16S T-RFLP, predicted T-RF sizes corresponded to Dehalococcoides sp., Geobacter spp. and Sulfurospirillum spp. restricted by AluI and Bsh1236I. In silico determined base pair length of the individual T-RF's are consistent with the actual T-RF size and previous results described by Mészáros et al. (2013). Based on the in silico data, the ratio of the Dehalococcoides sp. was usually above $5 \%$ in the bacterial community of the enrichments characterized with the presence of $\mathrm{VC}$ and ethene. The relative abundance of the genus Dehalococcoides (up to 18\%) showed significant but slow increase in the series of enrichments and the fermenters. Partial TCE degradation to cis-DCE was observed in enrichments characterized with the presence of Sulfurospirillum spp. (relative abundance up to $4.7 \%$ ) and/ or Geobacter spp. (relative abundance up to 29\%). However, the co-occurrence of the Sulfurospirillum spp. or Geobacter spp. was rarely detected.

\section{Discussion}

In our work two sites contaminated with short-chain chlorinated ethenes were selected to enrich organohalide-respiring microbial communities to produce site specific bioactive inocula at industrial scale. Groundwater samples originated from the selected contaminated sites were characterized with autochthonous organohalide-respiring microbial communities capable of complete dechlorination of PCE and TCE to harmless end-product ethene. Despite the presence of the Dehalococcoides sp. communities and of the three examined RDase genes, TCE was only partially dechlorinated to $c i s$-DCE at the non-treated site II suggesting the lack of nutrients for organohalide-respiring microorganisms. While at site I, treated with organic substrate, complete dechlorination of TCE to ethene was observed. Beyond the presence of organohalide-respiring microorganisms, available electron donors and nutrients are also needed for the complete dechlorination (Löffler and Edwards 2006). According to the presence and the activity of the organohalide-respiring bacteria, the collected groundwater samples proved to be appropriate to develop site-specific inocula. Effective dechlorinating microbial consortia were successfully enriched from groundwater samples collected from non-treated and biostimulated sites. The lag phase prior to detectable dechlorination activity in enrichments incubated at $8{ }^{\circ} \mathrm{C}$ in $500 \mathrm{~mL}$ volume and inoculated with microbial communities derived from different groundwater was usually observed within 30 days independently from their origin. Similar dechlorination performances with comparable time-scale were observed so far at higher temperature, above $12{ }^{\circ} \mathrm{C}$ (Cichocka et al. 2010; Löffler et al. 2013b; Mészáros et al. 2013). After the second and third transfer of the enrichments into higher $(2 \mathrm{~L}, 5 \mathrm{~L}, 10 \mathrm{~L}$ ) volumes along with TCE addition, the timecourse shortened with a progressively accelerated ability of dechlorination. During the serial transfer steps, enrichments inoculated with stimulated organohalide-respiring communities resulted in more extent and rapid dechlorination of TCE to cis-DCE compared to the enrichments inoculated with microbial community originated from the non-treated area. The applied biostimulation sufficiently enhanced the organohalide-respiring microorganisms and might have contributed to the accelerated dechlorination and the differently structured microbial communities in enrichments. Beyond the application of biostimulated microbial communities, the enhancement of microbial activity by sequential nutrient and vitamin addition (Mészáros et al. 2013) contributed to the efficient transfer from bench-scale to industrial-scale within less 18 months.

According to our results, Dehalococcoides sp. prevailing in the groundwater sustained in the enrichments even in those without ethene production. Complete dechlorination with the presence of $\mathrm{VC}$ and ethene was observed in enrichments characterized with the relative abundance of the Dehalococcoides sp. exceeding 5\%. In line with the accelerated transformation of the TCE, the relative abundance of Dehalococcoides sp. increased in the enrichments and the fermenters. Lee and Lee (2016) also found that the relative abundance of the Dehalococcoides sp. increased during the serial transfer of dechlorinating consortia. Moreover, they found that the complete dechlorination with $~ 1 \%$ relative abundance of Dehalococcoides sp. corresponded to $10^{8} 16 \mathrm{~S}$ rRNA gene copy $\mathrm{g}^{-1}$ (Lee and Lee 2016). In our enrichments, especially in fermenters, the total population of Dehalococcoides could be assumed equal or higher compared to Lee and Lee's results (2016). Considering the bioremediation of chlorinated ethenes contamination by bioaugmentation agents $\mathrm{Lu}$ et al. (2006) concluded that $10^{7}$ Dehalococcoides cells $\mathrm{L}^{-1}$ is necessary for the effective dechlorination. However, to achieve appropriate cell density on contaminated sites, up to several hundred litres of bioaugmentation agents should be used (Ellis et al. 2000; Vainberg et al. 2009; Pérez-de-Mora et al. 2014) depending on the Dehalococcoides cell density and the size of the contaminated field. 


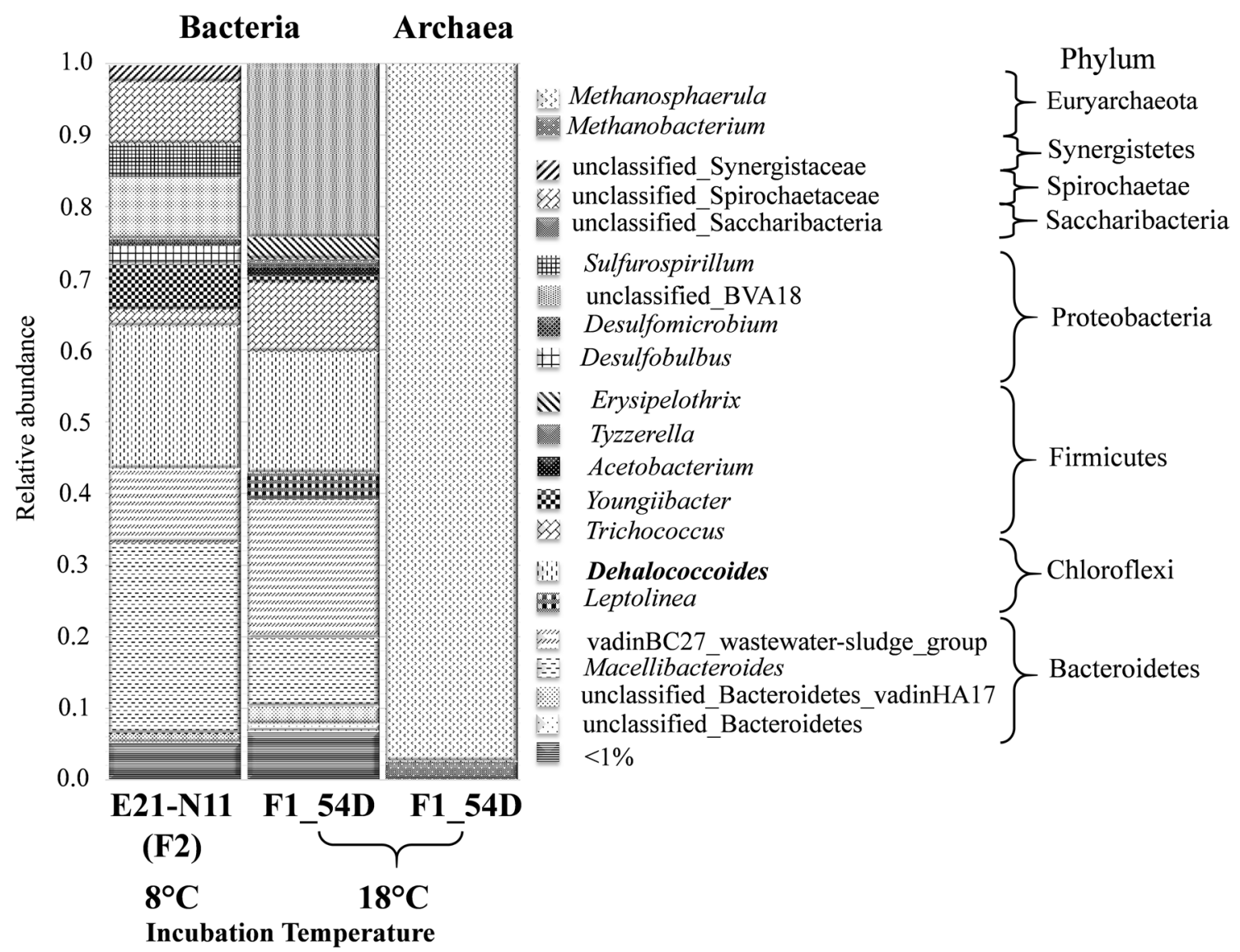

Fig. 4 Percentile distribution of amplicon sequences on order and phylum level revealed from fermenter F1 and composite sample of enrichments E21 and N11 used for inoculation of fermenter F2

According to the sequence analyses, different Dehalococcoides ribotypes affiliated with the Pinellas and Cornell subgroups were sustained in the enrichments differing regarding to electron acceptor utilization. The members of the Pinellas subgroup show highly similar 16S rRNA gene sequences with 1-3 nucleotide differences (Löffler et al. 2013a). To confirm the presence of the different Dehalococcoides sp. strains 16S rRNA gene sequencing was supplemented with the phylogenetic information of the RDase genes. The copresence of the three reductive dehalogenase genes suggests at least three different Dehalococcoides sp. strains in the fermenters and several enrichments. The different composition and ratio of the Dehalococcoides sp. strains could affect the biodegradation rate of the chlorinated ethenes that may have contributed to the observed differences in the dechlorination activity in fermenters. The accelerated dechlorination process without the accumulation of intermediates DCE and VC suggests the predominant presence of Dehalococcoides sp. BAV1 strain like microorganism (Cichocka et al. 2010) in the fermenter F2. The incomplete dechlorination to ethene with the periodic detection of the trans-DCE in the enrichment of fermenter F1 strengthens the presence of Dehalococcoides sp. CBDB1 strain like microorganism (Adrian et al. 2007). Besides the presence of the Dehalococcoides sp. CBDB1 strain like microorganism the dechlorination of trans-DCE without the accumulation of VC suggests the low ratio of the Dehalococcoides BAV1 strain like microorganism detected based on the catabolic $b v c A$ gene in the fermenter F1. With the presence of the Dehalococcoides sp. CBDB1 and BTF08 strains like microorganisms (Marco-Urrea et al. 2011; Cichocka et al. 2010) and Sulfurospirillum sp. (Luijten et al. 2003) in the fermenters F1 and F2, respectively, PCE dechlorination capability could be assumed in the enrichments of the fermenters. The revealed complex and diverse population of the different Dehalococcoides sp. strains resulted in the fastest TCE reduction to ethene in the enrichments of the different fermenters, which could be accelerated by the elevated temperature as well (Friis et al. 2007). However, based on the results of amplicon sequencing, the relative abundance of the Dehalococcoides sp. in the fermenter F1 was not higher than in composite enrichments used for inoculation of fermenter F2 incubated at $8{ }^{\circ} \mathrm{C}$. Considering the selective effect of the temperature, the presence and the relative abundance 
of the dominant members as Chloroflexi, Bacteroidetes and Firmicutes in enrichments incubated at different temperature showed similarities. Moreover, Bacteroidetes and Firmicutes were frequently detected in non-dechlorinating community of Dehalococcoides sp. dominated consortia incubated at temperature above $20^{\circ} \mathrm{C}$ (Ismaeil et al. 2017, 2018; Hug et al. 2012). Furthermore, the composite consortium (E21N11) incubated at $8{ }^{\circ} \mathrm{C}$ exhibited significant enrichment in $\delta$ - and $\varepsilon$-Proteobacteria, Spirochaetae and Synergistetes showing similarities to medium DonnalI incubated at $30^{\circ} \mathrm{C}$ (Hug et al. 2012). However, based on genus level, discrepancies were observed considering several fermentative bacteria. Trichococcus spp. (Liu et al. 2002), Youngiibacter spp. (Lawson et al. 2014) and Macellibacteroides spp. (Jabari et al. 2012) characterized with high relative abundance were present in the enrichment of the fermenter F1 and presumably originated from the sour whey. According to the presence and pronounced relative abundance of Saccharibacteria, the community composition of the enrichment in the fermenter F1 differed from that of formerly developed cultures (Duhamel et al. 2002; Waller et al. 2005; Duhamel and Edwards 2006; Holmes et al. 2006; Hug et al. 2012). Beside the different origin of the microbial community, the temperature and the surface provided by the solid phase, affected the settlement and the growth of microorganism inter alia the Dehalococcoides sp. strains. Overall, these parameters had effect on the composition of the organohalide-respiring microbial communities.

Fermentative bacteria can produce lactate, ethanol, hydrogen and acetate used by Dehalococcoides sp. as electron donors and carbon source, respectively. Lactate, amended in the enrichments by sour whey, can be transformed to acetate and hydrogen by Trichococcus sp. (Révész et al. 2006) and/or can be utilized as a carbon source and electron donor by sulphate-reducing bacteria as well. Similarly, to the observation of Révész et al. (2006) black precipitate was formed in the bottles indicating sulphide production that is indirect evidence for the activity of sulphate-reducing bacteria. The majority of the detected sulphate-reducing bacteria were acetogens implying intrinsic acetate production in the enrichments based on lactate and amino-acids. Fermentation products such as lactate, acetate and hydrogen support the growth of methanogenic Archaea beyond the organohalide-respiring microorganisms. Two members of the domain Archaea were identified with the significant prevalence of the hydrogenotrophic Methanosphaerula. To our knowledge this is the first time to detect the presence of Methanosphaerula in dechlorinating enrichments which are usually dominated by hydrogenotrophic Methanomicrobiales, Methanobacteriales, and acetotrophic Methanosarcinaceae and Methanoseataceae (Hug et al. 2012). Methanogenesis with increasing methane concentration co-occurred especially with accelerated dechlorination in the enrichment. The decreasing concentration of TCE and its intermediates may have contributed to the increase in methanogenesis, since PCE and its intermediate products such as TCE has inhibitory effect on methanogenic Archaea (DiStefano et al. 1991). However, several studies reported that dechlorination efficiency decreased in line with the accelerated methanogenesis (Aulenta et al. 2005; Men et al. 2013), since methanogens have a significant influence on the composition of the organohalide-respiring microbial communities (Men et al. 2013). According to our results the accelerated methanogenesis had no inhibitory effect on the dechlorination process. Presumably, hydrogen, lactate and acetate could be available in excess due to their addition and intrinsic formation by sour whey protein and biomass decay supporting the growth of dechlorinators and methanogens as well. Probably the availability of alternative electron donor such as lactate for dechlorinators contributed to the reduced competition between the organohalide-respiring and hydrogenotroph methanogen microorganisms. Methanogenic Archaea (Yan et al. 2013) along with the detected Geobacter spp., Sulfurospirillum spp., Desulfovibrio spp., Acetobacterium spp. and Clostridium spp. can produce corrinoid (Men et al. 2013) which is a preferred cofactor for Dehalococcoides sp. strains (Yan et al. 2013) growing on chlorinated ethenes. Similarly, developed enrichments maintained the metabolic functions important in supporting the Dehalococcoides sp. growth. This conserved metabolic profile with the presence of fermentative, acetogenic and methanogenic species contributed to complete and elevated dechlorination, which was maintained under low and high temperature as well. According to the dechlorination efficiency and the presence of the Dehalococcoides sp., the dechlorinating consortia maintained in fermenters of $100 \mathrm{~L}$ volume are suitable for performing pilot-scale tests conducted on site of the origin of organohalide-respiring communities.

\section{Conclusion}

Organohalide-respiring microbial communities were successfully enriched at laboratory- and industrial-scale fermenters. Microbial consortia derived from contaminated sites were used as seed culture characterized with differently structured autochthonous organohalide-respiring communities influenced by the availability of electron donors and nutrients. Nutrient addition on site and during cultivation (sequential transfer of enrichments into fresh media) enhanced the dechlorination process. Through serial transfer processes the detected diversity of Dehalococcoides sp. and its related RDase genes (tceA, $v c r A$ and $b v c A$ ) altered affecting the progress of dechlorination. To our knowledge this is the first attempt to enrich organohalide-respiring bacteria with accelerated dechlorination along with the increasing 
relative abundance of Dehalococcoides sp. in enrichments amended with clay mineral in pilot scale (upto $100 \mathrm{~L}$ ) at temperature below $20^{\circ} \mathrm{C}\left(8^{\circ} \mathrm{C}\right.$ and $\left.18{ }^{\circ} \mathrm{C}\right)$. Our results present cold adapted organohalide-respiring bacterial communities. In addition to the low temperature, the different origin of the microbial community and the solid-phase provided surface could contribute to the unique microbial composition of the developed consortia.

Acknowledgements Open access funding provided by Eötvös Loránd University (ELTE). This work was supported by Norway Grant (Project No. HU09-0046-A2-2013). The contribution of Balázs Vajna to the in silico analyses is gratefully acknowledged. The authors would like to thank for Tamás Felföldi for his assistance.

\section{Compliance with ethical standards}

Conflict of interest The authors declare that they have no conflict of interest.

Open Access This article is licensed under a Creative Commons Attribution 4.0 International License, which permits use, sharing, adaptation, distribution and reproduction in any medium or format, as long as you give appropriate credit to the original author(s) and the source, provide a link to the Creative Commons licence, and indicate if changes were made. The images or other third party material in this article are included in the article's Creative Commons licence, unless indicated otherwise in a credit line to the material. If material is not included in the article's Creative Commons licence and your intended use is not permitted by statutory regulation or exceeds the permitted use, you will need to obtain permission directly from the copyright holder. To view a copy of this licence, visit http://creativecommons.org/licenses/by/4.0/.

\section{References}

Adrian L, Hansen SK, Fung JM, Görisch H, Zinder SH (2007) Growth of Dehalococcoides strains with chlorophenols as electron acceptors. Envir Sci Tech 41(7):2318-2323

Aulenta F, Gossett JM, Rossetti PMP, Majone S (2005) Comparative study of methanol, butyrate, and hydrogen as electron donors for long-term dechlorination of tetrachloroethene in mixed anaerobic cultures. Biotechnol Bioeng 91(6):743-753

Cichocka D, Nikolausz M, Haest PJ, Nijenhuis I (2010) Tetrachloroethene conversion to ethene by a Dehalococcoides-containing enrichment culture from Bitterfeld. FEMS Microbiol Ecol 72(2):297-310

Culman SW, Bukowski R, Gauch HG, Cadillo-Quiroz H, Buckley DH (2009) T-REX: software for the processing and analysis of T-RFLP data. BMC Bioinformatics 10:171

Delgado AG, Fajardo-Williams D, Popat SC, Torres CI, Krajmalnik-Brown R (2014) Successful operation of continuous reactors at short retention times results in high-density, fast-rate Dehalococcoides dechlorinating cultures. Appl Microbiol Biot 98(6):2729-2737

DeWeerd KA, Mandelco L, Tanner RS, Woese CR, Suflita JM (1990) Desulfomonile tiedjei gen. nov. and sp. nov., a novel anaerobic, dehalogenating, sulfatereducing bacterium. Arch Microbiol 154:23-30

DiStefano TD, Gossett JM, Zinde SH (1991) Reductive dechlorination of high concentrations of tetrachloroethene to ethene by an anaerobic enrichment culture in the absence of methanogenesis. Appl Environ Microb 57(8):2287-2292

Duhamel M, Edwards EA (2006) Microbial composition of chlorinated ethene-degrading cultures dominated by Dehalococcoides. FEMS Microbiol Ecol 58(3):538-549

Duhamel M, Wehr SD, Yu L, Rizvi H, Seepersad D, Dworatzek S, Cox EE, Edwards EA (2002) Comparison of anaerobic dechlorinating enrichment cultures maintained on tetrachloroethene, trichloroethene, cis-dichloroethene and vinyl chloride. Water Res 36(17):4193-4202

El Fantroussi S, Mahillon J, Naveau H, Agathos SN (1997) Introduction of anaerobic microcosms and nested-PCR monitoring. Appl Environ Microb 63:806-811

Ellis DE, Lutz EJ, Odom JM, Buchanan RJ, Bartlett CL, Lee MD, Harkness MR, DeWeerd KA (2000) Bioaugmentation for accelerated in situ anaerobic bioremediation. Environ Sci Technol 34(11):2254-2260

Friis AK, Heimann AC, Jakobsen R, Albrechtsen HJ, Cox E, Bjerg PL (2007) Temperature dependence of anaerobic TCE-dechlorination in a highly enriched Dehalococcoides-containing culture. Water Res 41(2):355-364

Gerritse J, Renard V, Gomes TP, Lawson PA, Collins MD, Gottschal JC (1996) Desulfitobacterium sp. strain PCE1, an anaerobic bacterium that can grow by reductive dechlorination of tetrachloroethene or ortho-chlorinated phenols. Arch Microbiol 165(2):132-140

Hammer R, Harper DAT, Ryan PD (2001) Past paleontological statistics software package for education and data analysis. Palaeontol Electron. 4(1):9

He J, Sung Y, Krajmalnik-Brown R, Ritalahti KM, Löffler FE (2005) Isolation and characterization of Dehalococcoides sp. strain FL2, a trichloroethene (TCE)-and 1, 2-dichloroethene-respiring anaerobe. Environ Microbiol 7(9):1442-1450

Hendrickson ER, Payne JA, Young RM, Starr MG, Perry MP, Fahnestock S, Ellis DE, Ebersole RC (2002) Molecular analysis of Dehalococcoides $16 \mathrm{~S}$ ribosomal DNA from chloroethene-contaminated sites throughout North America and Europe. Appl Environ Microb 68:485-495

Holliger C, Hahn D, Harmsen H, Ludwig W, Schumacher W, Tindall B, Vazquez F, Weiss N, Zehnder AJ (1998) Dehalobacter restrictus gen. nov. and sp. nov., a strictly anaerobic bacterium that reductively dechlorinates tetra-and trichloroethene in an anaerobic respiration. Arch Microbiol 169(4):313-321

Holmes VF, He J, Lee PK, Alvarez-Cohen L (2006) Discrimination of multiple Dehalococcoides strains in a trichloroethene enrichment by quantification of their reductive dehalogenase genes. Appl Environ Microb 72(9):5877-5883

Hug LA, Beiko RG, Rowe AR, Richardson RE, Edwards EA (2012) Comparative metagenomics of three Dehalococcoides-containing enrichment cultures: the role of the non-dechlorinating community. BMC Genomics 13(1):327

Ismaeil M, Yoshida N, Katayama A (2017) Identification of multiple dehalogenase genes involved in tetrachloroethene-to-ethene dechlorination in a Dehalococcoides-dominated enrichment culture. BioMed Res Int. https://doi.org/10.1155/2017/9191086

Ismaeil M, Yoshida N, Katayama A (2018) Bacteroides sedimenti sp nov isolated from a chloroethenes-dechlorinating consortium enriched from river sediment. J Microbiol 56(9):619-627

Jabari L, Gannoun H, Cayol JL, Hedi A, Sakamoto M, Falsen E, Ohkuma M, Hamdi M, Fauque G, Ollivie B, Fardeau ML (2012) Macellibacteroides fermentans gen nov, sp. nov, a member of the family Porphyromonadaceae isolated from an upflow anaerobic filter treating abattoir wastewaters. Int J Syst Evol Microbiol 62(10), 2522-2527.

Klindworth A, Pruesse E, Schweer T, Peplies J, Quast C, Horn M, Glöckner FO (2013) Evaluation of general 16S ribosomal RNA 
gene PCR primers for classical and next-generation sequencingbased diversity studies. Nucleic Acids Res 41(1):e1. https://doi. org/10.1093/nar/gks808

Krajmalnik-Brown R, Hölscher T, Thomson IN, Saunders FM, Ritalahti KM, Löffler FE (2004) Genetic identification of a putative vinyl chloride reductase in Dehalococcoides sp. strain BAV1. Appl Environ Microb 70:6347-6351

Krett G, Szabó A, Felföldi T, Márialigeti K, Borsodi AK (2017) The effect of reconstruction works on planktonic bacterial diversity of a unique thermal lake revealed by cultivation, molecular cloning and next generation sequencing. Arch Microbiol 199(8):1077-1089

Krumholz LR (1997) Desulfuromonas chloroethenica sp. nov. uses tetrachloroethylene and trichloroethylene as electron acceptors. Int J Syst Bacteriol 47:1262-1263

Lane DJ (1991) 16S/23S rRNA sequencing. In: Stackebrandt E, Goodfellow $\mathrm{M}$ (eds) Nucleic acid techniques in bacterial systematics. Wiley, Chichester, pp 115-148

Lawson PA, Wawrik B, Allen TD, Johnson CN, Marks CR, Tanner RS, Harriman BH, Strąpoc D, Callaghan AV (2014) Youngiibacter fragilis gen. nov, sp. nov, isolated from natural gas production-water and reclassification of Acetivibrio multivorans as Youngiibacter multivorans comb. nov. Int J Syst Evol Microbiol 64(1):198-205

Lee J, Lee TK (2016) Development and characterization of PCE-toethene dechlorinating microcosms with contaminated river sediment. J Microbiol Biotechnol 26(1):120-129

Liu JR, Tanner RS, Schumann P, Weiss N, McKenzie CA, Janssen PH, Seviour EM, Lawson PA, Allen TD, Seviour RJ (2002) Emended description of the genus Trichococcus, description of Trichococcus collinsii sp. nov., and reclassification of Lactosphaera pasteurii as Trichococcus pasteurii comb. nov. and of Ruminococcus palustris as Trichococcus palustris comb nov in the low- $\mathrm{G}+\mathrm{C}$ Gram-positive bacteria. Int J Syst Evol Micr 52(4):1113-1126

Löffler FE, Edwards EA (2006) Harnessing microbial activities for environmental cleanup. Curr Opin Biotech 17(3):274-284

Löffler FE, Sun Q, Li J, Tiedje JM (2000) 16S rRNA gene-based detection of tetrachloroethene (PCE)-dechlorinating Desulfuromonas and Dehalococcoides species. Appl Environ Microb 66:1369-1374

Löffler FE, Ritalahti KM, Zinder SH (2013a) Dehalococcoides and reductive dechlorination of chlorinated solvents. In Stroo H, Leeson A, Ward C (eds) Bioaugmentation for groundwater remediation. SERDP ESTCP Environmental Remediation Technology. Springer, New York

Löffler FE, Yan J, Ritalahti KM, Adrian L, Edwards EA, Konstantinidis KT, Müller JA, Fullerton H, Zinder SH, Spormann AM (2013b) Dehalococcoides mccartyi gen. nov, sp. nov., obligately organohalide-respiring anaerobic bacteria relevant to halogen cycling and bioremediation, belong to a novel bacterial class, Dehalococcoidia classis nov., order Dehalococcoidales ord. nov. and family Dehalococcoidaceae fam. nov., within the phylum Chloroflexi. Int J Syst Evol Microbiol 63(2):625-635

Lu X, Wilson JT, Kampbell DH (2006) Relationship between Dehalococcoides DNA in ground water and rates of reductive dechlorination at field scale. Water Res 40(16):3131-3140

Luijten ML, de Weert J, Smidt H, Boschker HT, de Vos WM, Schraa G, Stams AJ (2003) Description of Sulfurospirillum halorespirans sp. nov., an anaerobic, tetrachloroethene-respiring bacterium, and transfer of Dehalospirillum multivorans to the genus Sulfurospirillum as Sulfurospirillum multivorans comb. nov. Int J Syst Evol Microbiol 53(3):787-793

Magnuson JK, Romine MF, Burris DR, Kingsley MT (2000) Trichloroethene reductive dehalogenase from Dehalococcoides ethenogenes: sequence of tceA and substrate range characterization. Appl Environ Microbiol 66:5141-5147
Marco-Urrea E, Nijenhuis I, Adrian L (2011) Transformation and carbon isotope fractionation of tetra-and trichloroethene to transdichloroethene by Dehalococcoides sp. strain CBDB1. Environ Sci Technol 45(4):1555-1562

Maymó-Gatell X, Chien YT, Gossett JM, Zinder SH (1997) Isolation of a bacterium that reductively dechlorinates tetrachloroethene to ethene. Science 276(5318):1568-1571

McCarty PL (1997) Breathing with chlorinated solvents. Science 276:1521-1522

Men Y, Lee PK, Harding KC, Alvarez-Cohen L (2013) Characterization of four TCE-dechlorinating microbial enrichments grown with different cobalamin stress and methanogenic conditions. Appl Microbiol Biotechnol 97(14):6439-6450

Mészáros É, Sipos R, Pál R, Cs R, Márialigeti K (2013) Stimulation of trichloroethene biodegradation in anaerobic three-phase microcosms. Int Biodeterior Biodegrad 84:126-133

Mohn WW, Tiedje JM (1990) Strain DCB-1 conserves energy for growth from reductive dechlorination coupled to formate oxidation. Arch Microbiol 153:267-271

Müller JA, Rosner BM, von Abendroth G, Meshulam-Simon G, McCarty PL, Spormann AM (2004) Molecular identification of the catabolic vinyl chloride reductase from Dehalococcoides sp. strain VS and its environmental distribution. Appl Environ Microbiol 70:4880-4888

Nagymáté Zs, Homonnay ZG, Márialigeti K (2016) Investigation of Archaeal and Bacterial community structure of five different small drinking water networks with special regard to the nitrifying microorganisms. Microbiol Res 188:80-89

Pérez-de-Mora A, Zila A, McMaster ML, Edwards EA (2014) Bioremediation of chlorinated ethenes in fractured bedrock and associated changes in dechlorinating and nondechlorinating microbial populations. Environ Sci Technol 48(10):5770-5779

Pöritz M, Goris T, Wubet T, Tarkka MT, Buscot F, Nijenhuis I, Lechne U, Adrian L (2013) Genome sequences of two dehalogenation specialists-Dehalococcoides mccartyi strains BTF08 and DCMB5 enriched from the highly polluted Bitterfeld region. FEMS Microbiol Lett 343(2):101-104

Pruesse E, Peplies J, Glöckner FO (2012) SINA: accurate highthroughput multiple sequence alignment of ribosomal RNA genes. Bioinformatics 28:1823-1829

Quast C, Pruesse E, Yilmaz P, Gerken J, Schweer T, Yarza P, Peplies J, Glöckner FO (2013) The SILVA ribosomal RNA gene database project: improved data processing and web-based tools. Nucleic Acids Res 41(D1):D590-D596

Révész S, Sipos R, Kende A, Rikker T, Romsics Cs, Mészáros É, Mohr A, Táncsics A, Márialigeti K(2006) Bacterial community changes in TCE biodegradation detected in microcosm experiments. Int Biodeterior Biodegr 58:239-247

Rowe AR, Lazar BJ, Morris RM, Richardson RE (2008) Characterization of the community structure of a dechlorinating mixed culture and comparisons of gene expression in planktonic and bioflocassociated "Dehalococcoides" and Methanospirillum species. Appl Environ Microbiol 74(21):6709-6719

Saiyari DM, Chuang HP, Senoro DB, Lin TF, Whang LM, Chiu YT, Chen YH (2018) A review in the current developments of genus Dehalococcoides, its consortia and kinetics for bioremediation options of contaminated groundwater. Sustain Environ Res 28(4):149-157

Schloss PD, Westcott SL, Ryabin T, Hall JR, Hartmann M, Hollister EB, Lesniewski RA, Oakley BB, Parks DH, Robinson CJ, Sahl JW, Stres B, Thallinger GG, Van Horn DJ, Weber CF (2009) Introducing mothur: open-source, platform-independent, communitysupported software for describing and comparing microbial communities. Appl Environ Microbiol 75:7537-7541

Smits THM, Devenoges C, Szynalski K, Maillard J, Holliger C (2004) Development of a real-time PCR method for quantification of the 
three genera Dehalobacter, Dehalococcoides, and Desulfitobacterium in microbial communities. J Microbiol Methods 57:369-378

Sung Y, Fletcher KE, Ritalahti KM, Apkarian RP, Ramos-Hernández N, Sanford RA, Mesbah NM, Löffler FE (2006) Geobacter lovleyi sp nov strain SZ, a novel metal-reducing and tetrachloroethene-dechlorinating bacterium. Appl Environ Microbiol 72(4):2775-2782

Szabó A, Korponai K, Kerepesi C, Somogyi B, Vörös L, Bartha D, Márialigeti K, Felföldi T (2017) Soda pans of the Pannonian steppe harbor unique bacterial communities adapted to multiple extreme conditions. Extremophiles 21(3):639-649

Tamura K, Stecher G, Peterson D, Filipski A, Kumar S (2013) MEGA6: Molecular evolutionary genetics analysis version 6.0. Mol Biol Evol 30:2725-2729. https://doi.org/10.1093/molbev/mst197

Taş N, Eekert V, Miriam HA, De Vos WM, Smidt H (2010) The little bacteria that can-diversity, genomics and ecophysiology of 'Dehalococcoides' spp. in contaminated environments. Microb Biotechnol 3(4):389-402

Tindall BJ, Rosselló-Mora R, Busse HJ, Ludwig W, Kämpfer P (2010) Notes on the characterization of prokaryote strains for taxonomic purposes. Int J Syst Evol Micr 60:249-266

Uchino Y, Miura T, Hosoyama A, Ohji S, Yamazoe A, Ito M, Ito Y, Suzuki K, Fujita N (2015) Complete genome sequencing of Dehalococcoides sp. strain UCH007 using a differential reads picking method. Stand Genomic Sci 10(1):102

Utkin I, Woese C, Wiegel J (1994) Isolation and characterization of Desulfitobacterium dehalogenans gen. nov., sp. nov., an anaerobic bacterium which reductively dechlorinates chlorophenolic compounds. Int J Syst Bacteriol 44:612-619
Vainberg S, Condee CW, Steffan RJ (2009) Large-scale production of bacterial consortia for remediation of chlorinated solvent-contaminated groundwater. J Ind Microbiol Biotechnol 36(9):1189-1197

Waller AS, Krajmalnik-Brown R, Löffler FE, Edwards EA (2005) Multiple reductive-dehalogenase-homologous genes are simultaneously transcribed during dechlorination by Dehalococcoidescontaining cultures. Appl Environ Microbiol 71(12):8257-8264

Wen LL, Zhang Y, Chen JX, Zhang ZX, Yi YY, Tang Y, Rittman BE, Zhao HP (2017) The dechlorination of TCE by a perchlorate reducing consortium. Chem Eng J 313:1215-1221

Yan J, Im J, Yang Y, Löffler FE (2013) Guided cobalamin biosynthesis supports Dehalococcoides mccartyi reductive dechlorination activity. Philos T R Soc B 368(1616):20120320

Zinder SH (1998) Methanogens. In: Burlage RS, Atlas R, Stahl D, Geesey G, Sayler G (eds) Techniques in microbial ecology. Oxford University Press, New York, pp 113-136

Ziv-El M, Delgado AG, Yao Y, Kang DW, Nelson KG, Halden RU, Krajmalnik-Brown R (2011) Development and characterization of DehaloR $^{\wedge} 2$, a novel anaerobic microbial consortium performing rapid dechlorination of TCE to ethene. Appl Microbiol Biotechnol 92(5):1063-1071

Publisher's Note Springer Nature remains neutral with regard to jurisdictional claims in published maps and institutional affiliations. 